\title{
La difícil decisión de ventilación mecánica en esclerosis lateral amiotrófica: A propósito de dos casos.
}

The difficult decision of mechanical ventilation in patients with Amyotrophic lateral sclerosis. Report of two cases.

REY DE CASTRO MUJICA Jorge ${ }^{1}$, PORTOCARRERO URDAY Alexandra ${ }^{2}$, VALDEZ FERNANDEZ-BACA Luis Manuel ${ }^{3}$.

\section{SUMMARY}

Amyotrophic Lateral Sclerosis (ALS) is a rapidly progressive neurological disease that attacks motor neurons responsible for controlling voluntary muscles. The cognitive functions of the patient remain intact. In the advanced stages the respiratory muscles fail and the patient needs some modality of ventilatory support. Two cases with ALS bulbar form diagnosis were benefited from this therapeutic intervention in our city.

KEY WORDS: ALS, ventilatory failure, mechanical ventilation.

\section{RESUMEN}

Esclerosis Lateral Amiotrófica (ELA) es una enfermedad progresiva y debilitante, invariablemente fatal que afecta a las neuronas motoras, en la que las funciones cognitivas del paciente permanecen intactas y habitualmente en las fases avanzadas se presenta insuficiencia respiratoria ventilatoria requiriendo alguna modalidad de soporte ventilatorio. Dos casos con diagnóstico de ELA forma bulbar se beneficiaron de esta intervención terapéutica en nuestra ciudad.

PALABRAS CLAVE: ELA, insuficiencia respiratoria, ventilación mecánica.

\footnotetext{
1 Neumólogo y Especialista en trastornos respiratorios del sueño. Clínica Anglo Americana. Profesor Principal de Medicina, Universidad Peruana Cayetano Heredia

Médico Asistente Clínica Anglo Americana.

Médico Internista e Infectólogo Clínica Anglo Americana. Profesor de Medicina, Universidad Peruana

Cayetano Heredia
} 


\section{INTRODUCCIÓN}

La Esclerosis Lateral Amiotrófica (ELA) es una enfermedad neuromuscular progresiva e irreversible de las motoneuronas, provocando debilidad y atrofia de la musculatura estriada. Es conocida en Estados Unidos como enfermedad de Lou Gehrig o Stephen Hawking, en Francia como Jean Martin Charcot o genéricamente como Enfermedad de Motoneuronas (EMN). Se estima que 20000 - 30000 americanos están afectados por la enfermedad con una tasa de incidencia anual de 2 casos por 100000 habitantes. El promedio de sobrevida es 3-5 años y en la fase final de la enfermedad el paciente habitualmente fallece en insuficiencia respiratoria ventilatoria. Dado que la patogenia asienta en las motoneuronas, el paciente mantiene la integridad absoluta del control de esfínteres, funciones neurocognitivas y sensoriales $(1,2,3)$. Presentamos dos casos de ELA en estadio avanzado de enfermedad que aceptaron ventilación mecánica convencional de por vida.

\section{Caso clínico 1}

Varón de 56 años. Antecedente de migraña y dislipidemia tratada con estatinas. Ex fumador de 1 paquete/día por 30 años, sin fumar hace cuatro años. Cuatro años atrás acudió a consulta por disartria, bradilalia, disfagia y fasciculaciones en la lengua. Progresivamente se presentaron fasciculaciones en piernas, muslos, abdomen, espalda, brazos, párpados y sialorrea. Dos años antes se le colocó sonda de gastrostomía vía endoscópica debido a franco deterioro de la deglución, ya se evidenciaba paresia de miembros inferiores. La electromiografía (EMG) cuatro años antes de su ingreso documentó actividad espontánea, potenciales de fasciculación y fibrilación en la lengua, miembros superiores e inferiores así como signos de reinervación, la velocidad de conducción era normal. Una segunda EMG hace dos años mostró lesión de neurona motora periférica y fasciculaciones. La resonancia magnética del encéfalo, el estudio de líquido céfalo raquídeo, pruebas de función hepática AST, ALT y DHL estaban dentro de valores normales y el estudio genético para enfermedad de Kennedy (mutación en el receptor de andrógeno Xq11-q12) fue negativo. Recibió tratamiento con clonazepam 0,5mg qd, amitriptilina $25 \mathrm{mg}$ qd y riluzole 50mg qd. Ingresó a emergencia por dificultad respiratoria, cianosis y cuadriparesia. En estas circunstancias difíciles, debido al evidente y severo compromiso de la ventilación, entrevistamos al paciente por primera vez. Al examen clínico estaba despierto, presentaba fasciculaciones en lengua, imposibilidad de articular palabra, muy pobre excursión torácica y uso de musculatura accesoria de la respira- ción del cuello, murmullo vesicular abolido en bases y roncantes en ambos campos pulmonares. PA 130/90 mmHg, FC 94x', FR $36 x^{\prime}, T^{\circ} 36,6^{\circ} \mathrm{C}$, SatO ${ }_{2} \mathrm{Hb} 93 \%$, $\left(\mathrm{FIO}_{2}\right.$ 0,32). La radiografía de tórax reveló atelectasias bibasales. Gases arteriales $\mathrm{pH} 7,38, \mathrm{pCO}_{2}$ 71,4, $\mathrm{pO}_{2}$ 56,4, $\mathrm{HCO}_{3} 39,8$ meq/L, $\mathrm{FiO}_{2}$ 0,4. Se le explicó al paciente y a la familia el nivel de deterioro funcional ventilatorio consecuencia de la enfermedad neurológica y la necesidad de considerar el uso de ventilación mecánica de por vida. Cuatro horas después el paciente asiente ingresar a ventilación mecánica. Es intubado y conectado a ventilador de inmediato. Cuarenta y ocho horas después se practica traqueostomía. Seis semanas después el paciente es dado de alta a domicilio donde previamente se implementaron las condiciones operativas necesarias para ventilación mecánica.

\section{Caso clínico 2}

Varón de 69 años, con antecedente de hemicolectomía por divertículos, ex fumador de 10 paquetes/año y sin fumar hace cinco años. Inició enfermedad hace tres años; en esa oportunidad fue evaluado ambulatoriamente debido a fasciculaciones y calambres en tórax, abdomen y miembros superiores e inferiores asociado a rigidez en los inferiores de ocho meses de evolución. Dos meses después, se agregó acúmulo de secreciones en cavidad oral, disfagia sobre todo con líquidos, disartria, disfonía y ausencia de reflejo nauseoso. Las fasciculaciones y debilidad muscular se incrementaron a predominio proximal en miembros superiores. Evolutivamente se evidenció atrofia de músculos de ambas manos e hiperreflexia osteo tendinosa. La espirometría cronometrada dos años atrás mostró: CVF 3,27 L (79,9 \%), VEF 12,57 L. (80,6 \%) y $\mathrm{VEF}_{1} / \mathrm{CVF}$ 78\%. Cinco meses después: CVF 2,55 L. (72\%), $\mathrm{VEF}_{1}$ 1,93 L. (69\%), $\mathrm{VEF}_{1} / \mathrm{CVF} 75 \%$. Una electromiografía veintisiete meses antes de su hospitalización documentó velocidad de conducción y potencial de acción normal, fasciculaciones y fibrilación en miembros inferiores. Seis meses después patrón de denervación en cuatro extremidades. Un tercer estudio cinco meses después mostró denervación miótomos cervical, toráxico y lumbar, velocidad de conducción cubital disminuida. La resonancia magnética del encéfalo y las pruebas de función hepática fueron normales. Un año antes del ingreso apareció disnea en reposo, ortopnea e insomnio de conciliación con imposibilidad de mantener la continuidad del sueño. No se pudo practicar espirometría cronometrada. Se instaló equipo Bi-level PAP con mascarilla de interfase nasal en modo espontáneo a presiones IPAP 14 y EPAP 5 lo que corrigió de inmediato el patrón de sueño y la ortopnea. Se instaló sonda de gastrostomía tres meses antes de la hospitalización debido al déficit en la deglución. Quin- 
ce días antes de su ingreso y en mejor condición clínica se explicó al paciente el curso de la enfermedad, la necesidad considerar la implementación de ventilación mecánica de por vida y las implicancias relacionadas a su calidad de vida, a lo que él accedió. Ingresó a emergencia por dificultad respiratoria, tos ineficiente y acúmulo de secreciones. Examen físico $\mathrm{SatO}_{2} \mathrm{Hb} 88 \%$ ( $\mathrm{FIO}_{2}$ 0,8), FC 122, FR $22 \mathrm{x}^{\prime}, \mathrm{PA}$ 160/95 mmHg, T $37,4^{\circ} \mathrm{C}$, murmullo vesicular ausente en bases de ambos campos pulmonares. La radiografía del tórax mostró atelectasias bibasales. $\mathrm{PO}_{2}$ 142,6 mmHg, $\mathrm{PCO}_{2} 40,1$ mmHg, pH 7,40, $\mathrm{HCO}_{3} 26,8$ meq/L, $\mathrm{CO}_{2} \mathrm{~T} 28,1$, EB 2,7, $\left(\mathrm{FiO}_{2}\right.$ 0,9). En esas condiciones se procedió a intubar y proporcionar ventilación mecánica. Veinticuatro horas después se realizó traqueostomía. El paciente fue dado de alta a su domicilio veintiocho días después con toda la infraestructura requerida para ventilación mecánica.

\section{DISCUSIÓN}

La ELA ha sido clasificada por la ICSD II (4) dentro de los trastornos del sueño por hipoventilacion/ hipoxemia secundarios a enfermedades neuromusculares. No afecta áreas del cerebro que intervienen en la regulación y alternancia del ciclo vigilia-sueño sin embargo el sueño puede verse afectado de manera indirecta por el movimiento periódico de los miembros asociado a micro despertares electroencefalográficos (arousals) y alteración respiratoria asociada. Esta última debido al compromiso del nervio frénico por la enfermedad y alteración en la función ventilatoria del diafragma. Característicamente la hipoventilación y desaturación se agravan durante el dormir y particularmente con sueño REM $(5,6)$. En estas condiciones es necesario que el paciente reciba los beneficios del soporte ventilatorio (7). En las fases precoces del trastorno respiratorio el paciente puede aquejar fatiga, somnolencia, sueño interrumpido e insomnio de conciliación $(8,9)$. Debe mencionarse también que la ansiedad, depresión, el dolor, las fasciculaciones, los calambres y las secreciones incrementadas pueden ser factores que no contribuyen a un buen dormir en estos pacientes $(10,11)$.

No hay un punto de corte preciso para definir cuando un paciente con ELA debe utilizar ventilación no invasiva. Son útiles clínicamente la fatiga, somnolencia diurna, despertares nocturnos frecuentes y el insomnio de conciliación. Se han propuesto variables espirométricas como la medición secuencial de la Capacidad Vital Forzada (CVF) y compararla en decúbito supino con posición sentado. Los registros espirométricos del caso 2 documentan el deterioro pro- gresivo de la CVF, la decisión se tomó en función de las dificultades que el paciente tenía para dormir. Otros procedimientos son las mediciones de la presión negativa intratoráxica y trans-diafragmática, del índice de desaturación por hora de sueño con oximetría nocturna y la implementación de polisomnografía precoz que permite objetivar el patrón de sueño, el esfuerzo respiratorio, la $\mathrm{SatO}_{2} \mathrm{Hb}$ e identificar transtornos respiratorios asociados $(1,7,12-15)$. Entre los equipos no invasivos están el CPAP y el Bi-level PAP en modo espontáneo. Estos se emplean para dormir y permiten que el paciente tenga una mejor calidad de vida y de sueño por un periodo que puede ir hasta un año como sucedió en el caso 2 (12,16-19).

Implementar ventilación mecánica convencional en pacientes con ELA va mucho más allá de cuestiones meramente técnicas. Díaz Lobato señala con claridad las condiciones requeridas para lograr el éxito en esta intervención. Es indispensable que el paciente este bien motivado y con deseos de vivir, y gozar de un estado de salud aceptable, aunque el autor no define este concepto. Debe apuntarse al mayor grado de autonomía posible sobretodo en la comunicación con el entorno. Tanto la familia como el paciente deben estar bien informados y conocer claramente los pros y contras de esta decisión en el contexto de una enfermedad paralizante, progresiva y fatal. La familia debe participar en el proceso y desear implementarlo. Es indispensable disponer de recursos económicos, técnicos y humanos (20). Estos requerimientos se cumplieron en ambos casos.

Según Moss AH y col, hasta $5 \%$ de los pacientes aceptan participar en esta modalidad terapéutica (21) y los que desisten son manejados de acuerdo a la presentación de los síntomas (22). Los enfermos deben conocer con antelación las alternativas terapéuticas para el manejo de la insuficiencia respiratoria, ello les permitirá procesar la información con tiempo y tomar una decisión en la mejor de sus condiciones físicas y sicológicas. No es recomendable hacerlo durante la crisis y bajo la presión de una muerte inminente. En esta circunstancia el principio de autonomía del paciente debe ser estrictamente respetado (7). La disyuntiva no debe plantearse como decisión correcta o incorrecta, ya que ello involucra el futuro del paciente en pleno uso de sus facultades y con conocimiento de lo que será una calidad de vida sustantivamente diferente.

Se desconoce la prevalencia de ELA en Perú. Hasta donde llega nuestra información siete pacientes con diagnóstico sólido de ELA, que incluyen los aquí presentados, reciben actualmente ventilación mecáni- 
ca convencional domiciliaria en la capital de la República.

El informe presenta el ingreso a ventilación mecánica de dos casos con ELA en un período de quince días en nuestra institución lo que constituye por si misma una experiencia enriquecedora que deseamos compartir con la comunidad médica.

\section{Correspondencia:}

Jorge Rey de Castro Mujica

Triana 375 - 5 Lima 21 Perú.

Correo electrónico: jorgerey@rcp.net.pe

Teléfono: 511-221-9013

\section{REFERENCIAS BIBLIOGRÁFICAS}

1. National Institute of Neurological Disorders and Stroke. Amyotrophic Lateral Sclerosis. Publicación de NIH 03-916. Junio 2002. En: http://www.ninds.nih.gov/ disorders/spanish/ esclerosis_lateral_amiotrofica.htm (Fecha de acceso: Abril del 2006).

2. Walling AD. Amyotrophic lateral sclerosis: Lou Gehrig’s Disease. Am Fam Physician 1999 ;59(6):148996.

3. Brooks BR. El Escorial World Federation of Neurology criteria for the diagnosis of amyotrophic lateral sclerosis. J Neurol Sci 1994;124:96-107.

4. American Academy of Sleep Medicine. The International Classification of sleep disorders. Second Edition. American Academy of Sleep Medicine; 2005. p. 76.

5. Bourke SC, Gibson GJ. Sleep and breathing in neuromuscular disease. Eur Resp J 2002;19: 1194 -1201.

6. Fergunson KA, Strong MJ, Ahmad D, et al. Sleepdisordered breathing in amyotrophic lateral sclerosis. Chest 1996;110:664-669.

7. National Quality Measures Clearinghouse. Practice parameter: the care of the patient with amyotrophic lateral sclerosis (an evidence-based review). Report of the Quality Standards Subcommittee of the American Academy of Neurology: ALS Practice Parameters Task Force. En: http://www.guideline.gov/ summary/summary.aspx?ss $=15 \&$ doc_id $=2819 \& \mathrm{nbr}=$ 2045. (Fecha de acceso: Abril del 2006).

8. Labanowsky M, Schmidt-Nowara W, Guilleminaud C. Sleep and neuromuscular disease: Frequency of sleed-disordered breathing in a neuromuscular disease clinic population. Neurology 1996;47:11731180.

9. Culebras A. Sleep disorders and neuromuscular disorders. En: Culebras A. Sleep disorders and neurological disease. New Cork: Marcel Dekker Inc; 2000. p. 402-403.

10. Charles G, Guilleminault C. Sleep and neuromuscular diseases. En: Kryger MH, Roth T, Dement WC. (Editores). Principles and practice of sleep medicine. Philadelphia: Elsevier Saunders; 2005. p. 833.

11. Chokroverty S. Sleep, breathing, and neurologic disorders. En: Chokroverti S, Daroff RB. Sleep disorders medicine: Basic science, technical considerations, and clinical aspects. Boston: Butterworth Heinemann; 1999. p. 531-532.

12. Kimura K, Tachibana N, Kimura J, Shibasaki H. Sleepdisordered breathing at an early stage of amyotrophic lateral sclerosis. J Neurol Sci 1999;15:1-2.

13. Kido M, Igase M, Nomura T, Kohara K, Miki T. Early treatment with non-invasive positive pressure ventilation a successful case of bulbar type amyotrophic lateral sclerosis. Nippon Ronen Igakkai Zasshi 2001; 38: 816-8.

14. Lechtzin N, Wiener CM, Clawson L, et al. Use of noninvasive ventilation in patients with amyotrophic lateral sclerosis. ALS \& Other Motor Neuron Disorders 2004; 5:9-15

15. Lyall RA, Donaldson N, Polkey MI, Leigh PN, Moxham J Respiratory muscle strength and ventilatory failure in amyotrophic lateral sclerosis. Brain 2001;124:200013.

16. Pinto AC, Evangelista T, Carvalho M, Alves MA, Sales L. Respiratory assistance with a non-invasive ventilator (Bipap) in MND/ALS patients: survival rates in a controlled trial. J Neurol Sci 1995;129:19-26.

17. Bach JR. Amyotrophic lateral sclerosis. Communication status and survival with ventilatory support. Am J Phys Med Rehabil 1994;73(3):218.

18. Golstein RS, Brooks D, Davis L. Sleep in patients with neuromuscular and chest wall disorders. En: McNicholas WT, Phillipson EA. Breathing disorders in sleep. London: WB Sounders; 2002. p. 310-321.

19. Krachman S, Criner GJ. Hypoventilation syndromes. En: Strollo PJ, Sanders MH. Sleep Disorders. Clinics in Chest Medicine 1998;19:152.

20. Salvador L. Soporte ventilatorio en los pacientes con ELA. En: http://neurologia.rediris.es/congreso-1/ conferencias/neuromuscular-2.html. (Fecha de acceso: Abril del 2006).

21. Moss AH, Casey P, Stocking CB, Roos RP, Brooks BR, Siegler M. Home ventilation for amyotrophic lateral sclerosis patients: outcomes, costs, and patient, family and physician attitudes. Neurology 1993;43:43843.

22. Ganzini L, Johnston W, Silveira M. The final month of life in patients with ALS. Neurology 2002;59:428-431.

Recibido: 29/05/06

Aceptado para publicación: 14/09/06 